\title{
Article
}

\section{A hybrid method for solution of linear Volterra integro-differential equations (LVIDES) via finite difference and Simpson's numerical methods (FDSM)}

\author{
Bashir Danladi Garba ${ }^{1,2, *}$ and Sirajo Lawan Bichi ${ }^{2}$ \\ 1 Department of Mathematics, Kano University of Science and technology, Wudil Kano, Nigeria. \\ 2 Department of Mathematical Sciences, Bayero University Kano, Nigeria. \\ * Correspondence: raposo@ufsj.edu.br \\ Communicated by: Absar ul Haq \\ Received: 9 August 2020; Accepted: 24 March 2021; Published: 27 May 2021.
}

\begin{abstract}
In this paper, a hybrid of Finite difference-Simpson's approach was applied to solve linear Volterra integro-differential equations. The method works efficiently great by reducing the problem into a system of linear algebraic equations. The numerical results shows the simplicity and effectiveness of the method, error estimation of the method is provided which shows that the method is of second order convergence.
\end{abstract}

Keywords: Linear Volterra integro-differential equation; Finite element method; Error estimation.

MSC: 65M50; 65M70; 65N35; 65M22.

\section{Introduction}

V ito Volterra in 1926 introduced integro-differential for the first time when he investigated the population growth, focussing his study on the hereditary influences, whereby through his research work the topic of integro-differential equations was established [1]. Mathematical modeling of real life problems often result in functional equations such as differential, integral and integro-differential equations. Many mathematical formulation of physical phenomena reduced to integro-differential equations like fluid dynamics, control theory, biological models and chemical kinetics [1-8].

Linear Integro-Differential Equation (LIDE) is an important branch of modern mathematics and arises often in many applied areas which include engineering, mechanics, physics, chemistry, astronomy, biology, economics, potential theory and electrostatics [9].

A variational iteration method and trapezoidal rule by Saadati et al., [10] was used for solving LIDEs. Manafianheris [11] applied modified laplace Adomian decomposition method. Bashir and Sirajo [12] used finite difference Simpson's approach on Fredholm Integro-differential equation and proved the error estimation of the method. In the work of Aruchunan and Sulaiman [2], a numerical solution of first order linear Fredholm integro-differential equations was obtained using conjugate gradient method. A reliable algorithm with application was presented by Alwaneh et al., [13]. Consider a linear Volterra integro-differential equation (LVIDE) of the form:

$$
u^{\prime}(x)=f(x)+\lambda \int_{a}^{x} k(x, t) u(t) d t, \quad u_{0}=\alpha,
$$

where $a$ and $\lambda$ are constants and $x$ is a variable, $f(x)$ and $k(x, t)$ are known function with $k(x, t)$ is the kernel and $u$ is the unknown function to be determined.

The main objective of this paper is to propose a combination of finite difference-Simpson's approach (FDSM) to solve (LVIDE) (1) by transforming the problem into a system of linear algebraic equations. We used Maple software to obtained the numerical solution. Some examples are given to test the accuracy and efficiency of the method.

The paper has been organized as follows; In Section 2, we presented the derivation of the method. Error estimation of the scheme is proved in Section 3 and numerical results and discussions are provided in Section 4. Finally Section 5 conclude the paper. 


\section{Derivation of the method}

Consider a linear Volterra integro-differential equation LVIDE of (1) and partition the domain $[a, x]$ into $N$ finite parts of uniform step length $h=\frac{x_{N}-a}{N}$, such that $x_{i}=a+i h, \quad i=1,2, \ldots, N$.

Now using composite Simpson's with $N$ subintervals. The integral part of (1) is approximated as:

$$
\begin{aligned}
\int_{a}^{x} k(x, t) u(t) d t \approx & \frac{h}{3}\left[k\left(x, t_{0}\right) u\left(t_{0}\right)+4\left(k\left(x, t_{1}\right) u\left(t_{1}\right)+\ldots+k\left(x, t_{N-1}\right) u\left(t_{N-1}\right)\right)\right. \\
& \left.+2\left(k\left(x, t_{2}\right) u\left(t_{2}\right)+\ldots+k\left(x, t_{N-2}\right) u\left(t_{N-2}\right)\right)+k\left(x, t_{N}\right) u\left(t_{N}\right)\right] \\
\approx & \frac{h}{3}\left[k\left(x, t_{0}\right) u\left(t_{0}\right)+4 k\left(x, t_{1}\right) u\left(t_{1}\right)+2 k\left(x, t_{2}\right) u\left(t_{2}\right)+\ldots+2 k\left(x, t_{N-2}\right) u\left(t_{N-2}\right)\right) \\
& \left.+4 k\left(x, t_{N-1}\right) u\left(t_{N-1}\right)+k\left(x, t_{N}\right) u\left(t_{N}\right)\right] .
\end{aligned}
$$

By discritizing along $x$ and taking $u^{\prime}\left(x_{i}\right)=u_{i}^{\prime}, f\left(x_{i}\right)=f_{i}, k\left(x_{i}, t_{i}\right)=k_{i j}, k\left(x_{i}, t_{i}\right)$ vanishes for $t_{j}>x_{i}$ we have

$$
u_{i}^{\prime}=f_{i}+\frac{h}{3}\left[k_{i 0} u_{0}+4 k_{i 1} u_{1}+2 k_{i 2} u_{2} \ldots+2 k_{i N 2} u_{N-2}+4 k_{i N-1} u_{N-1}+k_{i N} u_{N}\right]
$$

By central difference we can approximate the derivative part of (2) as

$$
u_{i}^{\prime}=\frac{u_{i+1}-u_{i-1}}{2 h}, \quad i=1,2, \ldots, N-1,
$$

and at the end point $N$ we use second Backward Finite difference

$$
u_{i}^{\prime}=\frac{3 u_{N}-4 u_{N-1}+u_{N-2}}{2 h}, \quad i=N
$$

By replacing $u_{i}^{\prime}$ in (2) we have

$$
\frac{u_{i+1}-u_{i-1}}{2 h}=f_{i}+\frac{h}{3}\left[k_{i 0} u_{0}+4 k_{i 1} u_{1}+2 k_{i 2} u_{2}+\ldots+2 k_{i N 2} u_{N-2}+4 k_{i N-1} u_{N-1}+k_{i N} u_{N}\right]
$$

for $i=1,2, \ldots, N-1$, and

$$
\begin{aligned}
\frac{3 u_{N}-4 u_{N-1}+u_{N-2}}{2 h}= & f_{N}+\frac{h}{3}\left[k_{N 0} u_{0}+4 k_{N 1} u_{1}+2 k_{N 2} u_{2}+\right. \\
& \left.\ldots+2 k_{N N 2} u_{N-2}+4 k_{N N-1} u_{N-1}+k_{N N} u_{N}\right]
\end{aligned}
$$

for $i=N$.

Using Equations (3) for $i=1,2, \ldots, N-1$ and (4) for $i=N$, we can generate a systems of linear equations for $u_{1}, u_{2}, \ldots, u_{N}$, which can be represented in a matrix form as $K U=W$, where

$$
K=\left(\begin{array}{cccccccc}
A_{11} & 1 & 0 & 0 & \ldots & 0 & 0 & 0 \\
A_{21}-1 & B_{22} & 1 & 0 & \ldots & 0 & 0 & 0 \\
A_{31}-1 & B_{32}-1 & A_{33} & 1 & \ldots & 0 & 0 & 0 \\
\cdot & \cdot & \cdot & \cdot & \cdot & \cdot & \cdot & \cdot \\
\cdot & \cdot & \cdot & \cdot & \cdot & \cdot & \cdot & \cdot \\
\cdot & \cdot & \cdot & \cdot & \cdot & \cdot & \cdot & . \\
A_{N-21} & B_{N-22} & A_{N-23} & B_{N-24} & \ldots & B_{N-2 N-2} & 1 & 0 \\
A_{N-11} & B_{N-12} & A_{N-13} & B_{N-14} & \ldots & B_{N-1 N-2}-1 & A_{N-1 N-1} & 1 \\
A_{N 1} & B_{N 2} & A_{N 3} & B_{N 4} & \ldots & B_{N N-2}+1 & A_{N N-1}-4 & C_{N N}+3
\end{array}\right)
$$




$$
U=\left(\begin{array}{c}
u_{1} \\
u_{2} \\
\cdot \\
\cdot \\
\cdot \\
u_{N}
\end{array}\right), \text { and } W=\left(\begin{array}{c}
2 h f_{1}+\left(\frac{2}{3} h^{2} k_{10}\right) u_{0} \\
2 h f_{2}+\frac{2}{3} h^{2} k_{20} u_{0} \\
\cdot \\
\cdot \\
2 h f_{N-1}+\frac{2}{3} h^{2} k_{N-10} u_{0} \\
2 h f_{N}+\frac{2}{3} h^{2} k_{N 0} u_{0}
\end{array}\right)
$$

\section{Error estimation}

Theorem 1. Suppose that $\mu_{1}, \mu_{2}, \mu_{3} \in(a, b)$ such that the errors $e_{1}$ of central difference, $e_{2}$ of second backward difference approximation and $e_{3}$ of Simpson's rule respectively are given by $\frac{h^{2}}{6} u^{(3)}\left(\mu_{1}\right), \frac{h^{2}}{4} u^{(4)}\left(\mu_{3}\right)$ and $\frac{\left(x_{N}-a\right)}{180} h^{4} u^{(4)}\left(\mu_{2}\right)$. Then the error estimation of approximate solution of linear Volterra integro-differential Equation (1) by the scheme (3) and (4) is $e^{*} \leq \frac{5\left(x_{N}-a\right)^{2}}{12 N^{2}} G$.

Proof. From the problems of LVIDES (3) and (4), the exact solution for $i=1,2, \ldots, N-1$

$$
\begin{aligned}
\frac{u_{i+1}-u_{i-1}}{2 h}+\frac{h^{2}}{6} u^{(3)}\left(\mu_{1}\right)= & f_{i}+\frac{h}{3}\left[k_{i 0} u_{0}+4 k_{i 1} u_{1}+2 k_{i 2} u_{2}+\ldots+2 k_{i N 2} u_{N-2}\right. \\
& \left.+4 k_{i N-1} u_{N-1}+k_{i N} u_{N}\right]+\frac{\left(x_{N}-a\right)}{180} h^{4} u^{(4)}\left(\mu_{2}\right),
\end{aligned}
$$

and for $i=N$

$$
\begin{aligned}
\frac{3 u_{N}-4 u_{N-1}+u_{N-2}}{2 h}+\frac{h^{2}}{4} u^{(4)}\left(\mu_{3}\right)= & f_{N}+\frac{h}{3}\left[k_{N 0} u_{0}+\ldots+2 k_{i N 2} u_{N-2}+4 k_{N N-1} u_{N-1}\right. \\
& \left.+k_{N N} u_{N}\right]+\frac{\left(x_{N}-a\right)}{180} h^{4} u^{(4)}\left(\mu_{2}\right)
\end{aligned}
$$

where $\mu_{1}, \mu_{2}, \mu_{3} \in(a, b)$.

Subtracting (3) and (4) from (5) and (6), we obtained the error term as follow:

$$
\begin{aligned}
e & =\left|\frac{h^{2}}{6} u^{(3)}\left(\mu_{1}\right)+\frac{h^{2}}{4} u^{(4)}\left(\mu_{3}\right)-\left(\frac{\left(x_{N}-a\right)}{180} h^{4} u^{(4)}\left(\mu_{2}\right)+\frac{\left(x_{N}-a\right)}{180} h^{4} u^{(4)}\left(\mu_{2}\right)\right)\right|, \\
& =\left|\frac{h^{2}}{6} u^{(3)}\left(\mu_{1}\right)+\frac{h^{2}}{4} u^{(4)}\left(\mu_{3}\right)-\frac{\left(x_{N}-a\right)}{90} h^{4} u^{(4)}\left(\mu_{2}\right)\right|, \\
& \leq\left|\frac{h^{2}}{6} u^{(3)}\left(\mu_{1}\right)+\frac{h^{2}}{4} u^{(4)}\left(\mu_{3}\right)\right|, \\
& \leq\left|\frac{h^{2}}{6} G_{1}+\frac{h^{2}}{4} G_{2}\right|
\end{aligned}
$$

after taking $G_{1}=u^{(3)}\left(\mu_{1}\right)$ and $G_{2}=u^{(4)}\left(\mu_{3}\right)$.

Now, if we let $G=\max \left\{G_{1}, G_{2}\right\}$ we have

$$
e \leq\left|\frac{h^{2}}{6} G+\frac{h^{2}}{4} G\right|, \leq\left|\frac{5 h^{2}}{12} G\right| .
$$

Substituting $h=\frac{x_{N}-a}{N}$, we get

$$
e^{*} \leq\left|\frac{5\left(x_{N}-a\right)^{2}}{12 N^{2}} G\right| .
$$

Hence the proposed scheme is of second order convergence.

\section{Results and discussion}

In this section the proposed method of finite difference-Simpson's approach is used to obtain the numerical solutions of problems of LVIDES in order to study the performance of the method. 
Example 1. Consider LVIDE equation:

$$
u^{\prime}(x)=1+\sin x+\int_{0}^{x} u(t) d t, \quad u(0)=-1,
$$

with exact solution $u(x)=\frac{e^{x}}{4}-\frac{3 e^{-x}}{4}-\frac{\cos x}{2}$. We obtain the numerical results at different values of $x$ which are represented in Table 1:

Table 1. Numerical result of Example 1

\begin{tabular}{c|c|c|c}
$x$ & Exact solution & FDSM & REA[13] \\
\hline 0.0625 & -0.9374599377 & -0.970285632 & -0.939313 \\
\hline 0.125 & -0.8746843974 & -0.881205977 & -0.877167 \\
\hline 0.1875 & -0.8114509330 & -0.848304304 & -0.816836 \\
\hline 0.250 & -0.7475504439 & -0.760345075 & -0.752159 \\
\hline 0.3125 & -0.6827862105 & -0.723778451 & -0.691295 \\
\hline 0.375 & -0.6169729163 & -0.635854259 & -0.623507 \\
\hline 0.43750 & -0.5499356617 & -0.595245125 & -0.561194 \\
\hline 0.500 & -0.4815089580 & -0.506345938 & -0.489657 \\
\hline 0.5625 & -0.4115357039 & -0.461405361 & -0.425194 \\
\hline 0.6250 & -0.3398661420 & -0.370577838 & -0.349485 \\
\hline 0.6875 & -0.2663567891 & -0.316674778 & -0.282077 \\
\hline 0.750 & -0.1908693447 & -0.231335606 & -0.201611 \\
\hline 0.8125 & -0.1132695669 & -0.165028649 & -0.130719 \\
\hline 0.8750 & -0.0334261204 & -0.08294371 & -0.0451471 \\
\hline 0.93750 & 0.0487906069 & -0.005464919 & -0.0299532 \\
\hline 1 & 0.1335097231 & 0.075642841 & -0.121113
\end{tabular}

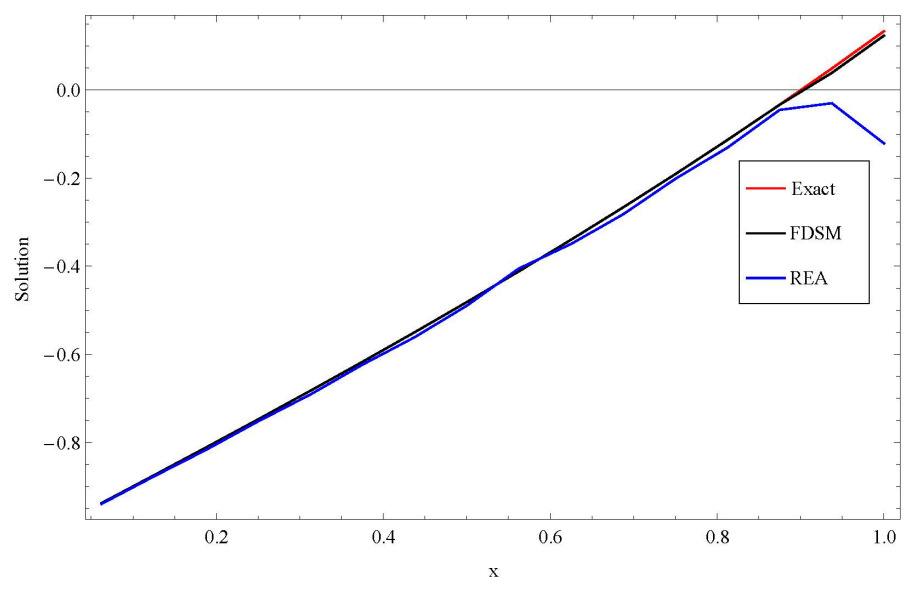

Figure 1. Comparison of FDSM and REA

Table 1 shows the numerical results of Example 1. We compare the results obtained by using our method and numerical method of Romberg extrapolation algorithm (REA) given in [13]. Figure 1 indicate that our method of FDSM coincide with the exact solution and gives a better result than REA. This prove that our method is a good tool of approximating LVIDES problems of second kind.

Example 2. Consider LVIDE equation:

$$
u^{\prime}(x)=-\sin x-\cos x+2 \int_{0}^{x} \cos (x-t) u(t) d t, \quad u(0)=1
$$

with exact solution $u(x)=e^{-x}$. The numerical results are represented in Table 2 below: 
Table 2. Numerical result of Example 2

\begin{tabular}{c|c|c|c}
$x$ & Exact solution & FDSM & BVMs[14] \\
\hline 0.1 & 0.7788007831 & 0.6913562245 & 0.5627307831 \\
\hline 0.2 & 0.6065306597 & 0.5986143128 & 0.5781196597 \\
\hline 0.3 & 0.4723665527 & 0.0467629062 & 0.4687287527 \\
\hline 0.4 & 0.3678794412 & 0.3678699844 & 0.3674183412
\end{tabular}

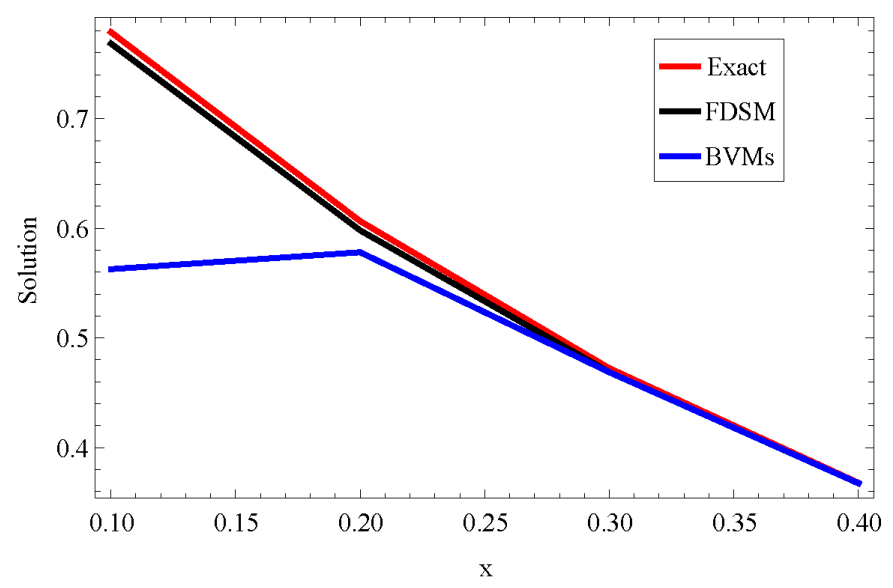

Figure 2. Comparison of FDSM and BVMs

Table 2 shows the exact and the approximate solution obtained by our method at different values of $x$ with the results of Boundary value methods (BVMs) given in [14]. Figure 2 provided a graphical presentation of the result which shows that our method can converge to the exact solution and gives a better result than BVMs.

Example 3. Consider LVIDE equation:

$$
u^{\prime}(x)=1-\int_{0}^{x} u(t) d t, \quad u(0)=0
$$

with exact solution $u(x)=\sin x$. The numerical results are represented in Table 3 .

Table 3. The exact and approximate solution of Example 3

\begin{tabular}{c|c|c|c}
$x$ & Exact solution & FDSM & Absolute error \\
\hline 0.1 & 0.09983341665 & 0.0966907300 & $3.143 e-3$ \\
\hline 0.2 & 0.1986693308 & 0.2009669073 & $2.298 e-3$ \\
\hline 0.3 & 0.2955202067 & 0.3006342060 & $5.114 e-3$ \\
\hline 0.4 & 0.3894183423 & 0.4099264019 & $2.051 e-2$ \\
\hline 0.5 & 0.4794255386 & 0.5166993051 & $3.727 e-2$ \\
\hline 0.6 & 0.5646424734 & 0.6352577593 & $7.061 e-2$ \\
\hline 0.7 & 0.6442176872 & 0.7535502344 & $1.093 e-1$ \\
\hline 0.8 & 0.7173560909 & 0.8859967676 & $1.686 e-1$ \\
\hline 0.9 & 0.7833269096 & 1.020684713 & $2.374 e-1$ \\
\hline 1 & 0.8414709848 & 1.172198061 & $3.307 e-1$
\end{tabular}




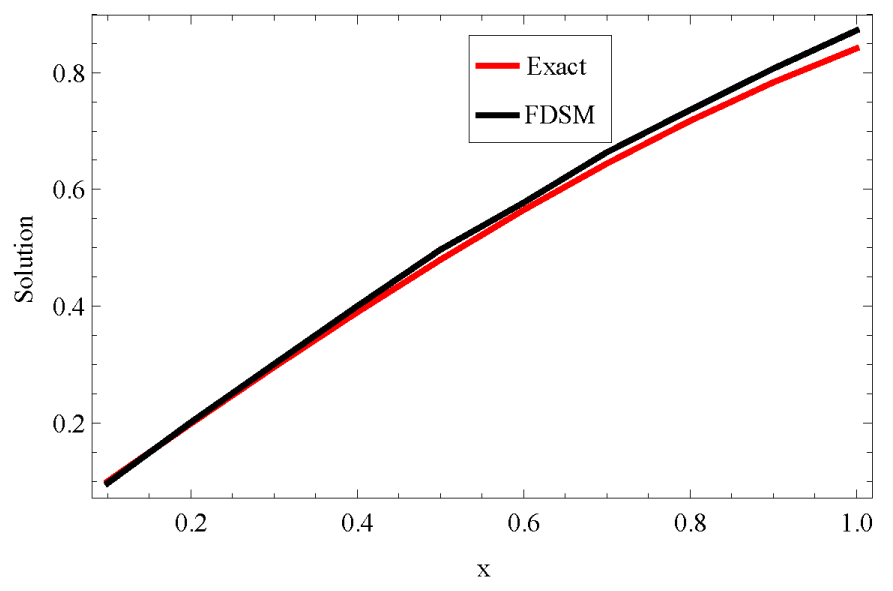

Figure 3. Graph of numerical result when solving Example 3

Table 3 shows the exact solution of the problem in Example 3 and the approximate solution obtained by our method. The absolute error obtained indicated that our method can give good approximation to LVIDE problems.

\section{Conclusion}

In this paper, we presented a numerical method to solve problems of linear Volterra integro-differential equations using a finite difference-Simpson's approach by transforming the problem into a system of linear algebraic equations. Error estimation of the method shows that the method has second order convergence. Some problems and comparison with two method of REA and BVMs is given in order to test the applicability and efficiency of the derived method which prove that our method converges to the exact solution.

Acknowledgments: The authors are grateful to the referees for their useful comments.

Author Contributions: All authors contributed equally to the writing of this paper. All authors read and approved the final manuscript.

Conflicts of Interest: "The author declares no conflict of interest."

\section{References}

[1] Isler Acar, N., \& Dascioglu, A. (2019). A projection method for linear FredholmŰVolterra integro-differential equations. Journal of Taibah University for Science, 13(1), 644-650.

[2] Darania, P., \& Ebadian, A. (2007). A method for the numerical solution of the integro-differential equations. Applied Mathematics and Computation, 188(1), 657-668.

[3] Danfu, H., \& Xufeng, S. (2007). Numerical solution of integro-differential equations by using CAS wavelet operational matrix of integration. Applied Mathematics and Computation, 194(2), 460-466.

[4] Vahidi, A. R., Babolian, E., Cordshooli, G. A., \& Azimzadeh, Z. (2009). Numerical solution of Fredholm integro-differential equation by AdomianŠs decomposition method. International Journal of Mathematical Analysis, 3(33-36), 1769-1773.

[5] Hosseini, S. M., \& Shahmorad, S. (2005). Numerical piecewise approximate solution of Fredholm integro-differential equations by the Tau method. Applied Mathematical Modelling, 29(11), 1005-1021.

[6] Raftari, B. (2010). Numerical solutions of the linear volterra integro-differential equations: homotopy perturbation method and finite Difference method. World Applied Sciences Journal, 9, 7-12.

[7] Tamamagar, M. (2014). The Numerical Solution of linear Fredholm integro-differential equations via Parametric iteration method. Applied and Computational Mathematics, 3, 4-10.

[8] Pandey, P. K. (2015). Numerical Solution of Linear Fredholm Integro-Differential Equations by Non-Standard Finite Difference Method. International Journal of Computer Mathematics, 5, 259-266.

[9] Kurt, N., \& Sezer, M. (2008). Polynomial solution of high-order linear Fredholm integro-differential equations with constant coefficients. Journal of the Franklin Institute, 345(8), 839-850. 
[10] Saadati, R., Raftari, B., Abibi, H., Vaezpour, S. M., \& Shakeri, S. (2008). A comparison between the Variational Iteration method and Trapezoidal rule for solving linear integro-differential equations. World Applied Sciences Journal, 4(3), 321-325.

[11] Heris, J. M. (2012). Solving the integro-differential equations using the modified Laplace Adomian decomposition method. Journal of Mathematical Extension, 6(1), 41-55.

[12] Garba, B. D., \& Bichi, S. L. (2020). On solving linear Fredholm integro-differential equations via finite difference-SimpsonŠs approach. Malaya Journal of Matematik (MJM), 8(2), 469-472.

[13] Al-Towaiq, M., \& Kasasbeh, A. (2017). Modified Algorithm for Solving Linear Integro-Differential Equations of the Second Kind. American Journal of Computational Mathematics, 7(02), 157-165.

[14] Chen, H., \& Zhang, C. (2011). Boundary value methods for Volterra integral and integro-differential equations. Applied Mathematics and Computation, 218(6), 2619-2630.

(C) 2021 by the authors; licensee PSRP, Lahore, Pakistan. This article is an open access article distributed under the terms and conditions of the Creative Commons Attribution (CC-BY) license (http://creativecommons.org/licenses/by/4.0/). 